\title{
LOCAL SAMPLING THEOREMS FOR SPACES GENERATED BY SPLINES WITH ARBITRARY KNOTS
}

\author{
WENCHANG SUN
}

\begin{abstract}
Most of the known results on sampling theorems, e.g., regular and irregular sampling theorems for band-limited functions, are concerned with global sampling. That is, to recover a function at a point or on an interval, we have to know all the samples, which are usually infinitely many. On the other hand, local sampling, which invokes only finitely many samples to reconstruct a function on a bounded interval, is practically useful since we only need to consider a function on a bounded interval in many cases and hardware can process only finitely many samples. In this paper, we give a characterization of local sampling sequences for spaces generated by B-splines with arbitrary knots.
\end{abstract}

\section{INTRODUCTION AND MAIN RESULTS}

One of the main applications of sampling theorems is to reconstruct functions from sampled values. Specifically, for a certain function space $V$ and a sequence of sampling points $\left\{x_{k}: k \in \mathbb{Z}\right\}$, there is a sequence of functions $\left\{S_{k}(x): k \in \mathbb{Z}\right\} \subset V$ such that for any $f \in V$,

$$
f(x)=\sum_{k \in \mathbb{Z}} f\left(x_{k}\right) S_{k}(x) .
$$

The sampling theorem is one of the most powerful tools in signal analysis and it is therefore very useful to characterize sampling sequences for a given function space. For the space of band-limited functions, the theory is well understood by the work of Beurling [5], Landau [15], Jaffard [13, Ortega-Cerdà and Seip [18, and several others. For the sampling on general shift invariant subspaces, however, many questions remain.

Note that in many cases (e.g., in the Shannon sampling theorem for band-limited functions [20] and many other sampling theorems for shift invariant subspaces 1 , [2, 3, 4, 6, 10, 12, 16]), $S_{k}$ is not compactly supported. That means, to recover the function at a point or on a finite interval, we have to know all the (infinitely many) samples. On the other hand, since hardware can handle only finitely many

Received by the editor December 6, 2006 and, in revised form, October 25, 2007.

2000 Mathematics Subject Classification. Primary 65T40, Secondary 41A58, 42A65, 42C15, $94 \mathrm{~A} 20$

Key words and phrases. Local sampling theorems, local sampling sequences, spline subspaces, irregular sampling, periodic nonuniform sampling.

This work was supported partially by the National Natural Science Foundation of China (10571089 and 60472042), the Program for New Century Excellent Talents in Universities, and the Scientific Research Foundation for the Returned Overseas Chinese Scholars, State Education Ministry. 
data, it is practically useful to consider local sampling. That is, given a function space $V$ and an interval $[a, b]$, we want to find conditions on the sampling sequence $\left\{x_{k}: 0 \leq k \leq K-1\right\} \subset[a, b]$ such that there is a sequence of functions $\left\{S_{k}: 0 \leq\right.$ $k \leq K-1\}$ with

$$
f(x)=\sum_{k=0}^{K-1} f\left(x_{k}\right) S_{k}(x), \quad \forall f \in V, x \in[a, b] .
$$

As far as we know, the only result in this aspect is the periodic nonuniform sampling theorem for cardinal B-spline subspaces. Specifically, let $W_{m}$ be the shift invariant subspace generated by the cardinal B-spline $\chi_{[0,1]} * \cdots * \chi_{[0,1]}(m+1$ terms) and let $0 \leq x_{0}<x_{1}<\cdots<x_{m}<1$ be fixed. Then we can find compactly supported $S_{0}, S_{1}, \ldots, S_{m} \in W_{m}$ such that

$$
f(x)=\sum_{k \in \mathbb{Z}} \sum_{0 \leq p \leq m} f\left(x_{p}+k(m+1)\right) S_{p}(x-k(m+1)), \quad \forall f \in W_{m} .
$$

In this case, $f(x)$ is determined by finitely many samples near $x$. However, the distribution of sampling points is bad. There are $m+1$ sampling points in every interval such as $[k(m+1), k(m+1)+1)$ while there is no sampling point in the subsequent intervals of length $m$.

In [24], the authors studied local sampling problems on spaces generated by cardinal B-splines and gave a characterization of local sampling sequences for these spaces. In this paper, we consider the same problem but with more general settings. Specifically, we consider spline spaces with arbitrary knot sequences.

Let $\Gamma=\left\{t_{k}: k \in \mathbb{Z}\right\}$ be a sequence of real numbers such that

$$
t_{k} \leq t_{k+1} \quad \text { and } \quad t_{k}<t_{k+m}, \quad k \in \mathbb{Z},
$$

where $m \geq 1$ is a fixed integer. Note that the above inequalities show that each point can appear at most $m$ times in the sequence. Let $\varphi_{n}$ be the $m$-degree B-spline with knots $\left(t_{n}, t_{n+1}, \ldots, t_{n+m+1}\right)$ and

$$
V_{m}=\left\{\sum_{n \in \mathbb{Z}} c_{n} \varphi_{n}: c_{n} \in \mathbb{C}\right\} .
$$

Observe that $V_{m}$ stands for a large class of function spaces. For example, for $\Gamma=\mathbb{Z}, \varphi_{n}$ is exactly the $m$-degree cardinal B-spline, i.e., $\varphi_{n}=B_{m}(\cdot-n)$, where $B_{m}=\chi_{[0,1]} * \cdots * \chi_{[0,1]}(m+1$ terms $)$.

Another interesting example is $\Gamma=\{\lfloor n / r\rfloor: n \in \mathbb{Z}\}$, where $1 \leq r \leq m$ and we use the notation $\lfloor x\rfloor:=\max \{n \in \mathbb{Z}: n \leq x\}$. In this case,

$$
V_{m}^{(r)}=\left\{\sum_{n \in \mathbb{Z}, 1 \leq l \leq r} c_{n, l} \psi_{l}(\cdot-n): c_{l, n} \in \mathbb{C}\right\},
$$

where $\psi_{l}$ is the normalized B-spline with knots $(\lfloor l / r\rfloor,\lfloor(l+1) / r\rfloor, \ldots$, $\lfloor(l+m+1) / r\rfloor)$. These splines are investigated in the study of wavelets of multiplicity $r$. We refer to [11, 17] for details.

For convenience, we introduce the following definitions. Given a knot sequence $\left\{t_{k}: k \in \mathbb{Z}\right\}$, we define

$$
\begin{aligned}
L(k) & :=L_{\Gamma}(k)=\min \left\{k^{\prime} \leq k: t_{k^{\prime}}=t_{k}\right\} \\
R(k) & :=R_{\Gamma}(k)=\max \left\{k^{\prime} \geq k: t_{k^{\prime}}=t_{k}\right\}
\end{aligned}
$$


For simplicity, we write $L(k)$ and $R(k)$ instead of $L_{\Gamma}(k)$ and $R_{\Gamma}(k)$, respectively. It is easy to see that $t_{k}$ appears exactly $R(k)-L(k)+1$ times in $\Gamma$. By (1.1), we have

$$
0 \leq R(k)-L(k) \leq m-1, \quad \forall k \in \mathbb{Z} .
$$

Let \#E denote the cardinality of a sequence $E$.

Definition 1.1. We call a sequence $E$ an $m$-spread sequence on $\left[t_{N_{1}}, t_{N_{2}}\right]$ with respect to $\left\{t_{k}: k \in \mathbb{Z}\right\}$ if $E \subset\left[t_{N_{1}}, t_{N_{2}}\right]$ consists of distinct points and satisfies the following:

$$
\begin{aligned}
& \# E \geq L\left(N_{2}\right)-R\left(N_{1}\right)+m, \\
& \#\left(E \cap\left[t_{N_{1}}, t_{n}\right)\right) \geq R(n)-R\left(N_{1}\right), \quad N_{1} \leq n \leq N_{2}, \\
& \#\left(E \cap\left(t_{n}, t_{N_{2}}\right]\right) \geq L\left(N_{2}\right)-L(n), \quad N_{1} \leq n \leq N_{2}, \\
& \#\left(E \cap\left(t_{n_{1}}, t_{n_{2}}\right)\right) \geq R\left(n_{2}\right)-L\left(n_{1}\right)-m, \quad N_{1} \leq n_{1} \leq n_{2} \leq N_{2} .
\end{aligned}
$$

Definition 1.2. We call a sequence $E:=\left\{x_{k}: 0 \leq k \leq K-1\right\}$ a local sampling sequence for $V_{m}$ on $\left[t_{N_{1}}, t_{N_{2}}\right]$ if $E \subset\left[t_{N_{1}}, t_{N_{2}}\right]$, and there is a sequence of functions $\left\{S_{k}: 0 \leq k \leq K-1\right\}$ such that

$$
f(x)=\sum_{k=0}^{K-1} f\left(x_{k}\right) S_{k}(x), \quad \forall f \in V_{m}, x \in\left[t_{N_{1}}, t_{N_{2}}\right] .
$$

We only need to consider local sampling sequences consisting of distinct points. In fact, if $E$ is a local sampling sequence, then the largest subsequence of $E$ which contains only distinct points is also a local sampling sequence and vice visa.

Note that there is not a local sampling sequence for band-limited functions. In fact, since a band-limited function is the restriction of an analytical function on the real line, if $f(x)=\sum_{k=0}^{K-1} f\left(x_{k}\right) S_{k}(x)$ holds on some interval, then $f$ is determined on the whole real line, which is impossible since a band-limited function is not determined by finitely many samples in general.

For the case of spaces generated by B-splines, however, such a local sampling sequence does exist. In fact, we have the following.

Theorem 1.1. A sequence of distinct points is a local sampling sequence for $V_{m}$ on $\left[t_{N_{1}}, t_{N_{2}}\right]$ if and only if it is an $m$-spread sequence with respect to $\left\{t_{k}: k \in \mathbb{Z}\right\}$ on the same interval.

By setting $t_{n}=\lfloor n / r\rfloor$, we get a characterization of local sampling sequences for the space $V_{m}^{(r)}$ defined by (1.2).

Corollary 1.2. A sequence of distinct points is a local sampling sequence for $V_{m}^{(r)}$ on $\left[N_{1}^{\prime}, N_{2}^{\prime}\right]$ if and only if it satisfies the following:

$$
\begin{aligned}
& \# E \geq r\left(N_{2}^{\prime}-N_{1}^{\prime}-1\right)+m+1, \\
& \#\left(E \cap\left[N_{1}^{\prime}, N_{1}^{\prime}+k\right)\right) \geq r k, \quad 0 \leq k \leq N_{2}^{\prime}-N_{1}^{\prime}, \\
& \#\left(E \cap\left(N_{2}^{\prime}-k, N_{2}^{\prime}\right]\right) \geq r k, \quad 0 \leq k \leq N_{2}^{\prime}-N_{1}^{\prime}, \\
& \#\left(E \cap\left(n_{1}^{\prime}, n_{2}^{\prime}\right)\right) \geq r\left(n_{2}^{\prime}-n_{1}^{\prime}+1\right)-m-1, \quad N_{1}^{\prime} \leq n_{1}^{\prime} \leq n_{2}^{\prime} \leq N_{2}^{\prime} .
\end{aligned}
$$

By setting $r=1$, we get 24, Theorem 1.1].

We give the proof of Theorem 1.1 in Section 2. As applications, we give several local sampling theorems for $V_{m}^{(r)}$ in Section 3 . 


\section{Proof OF MAIN RESUlts}

We begin with a result on $m$-spread sequences.

Lemma 2.1. Let $E$ be an m-spread sequence on $\left[t_{N_{1}}, t_{N_{2}}\right]$ with respect to $\left\{t_{k}: k \in\right.$ $\mathbb{Z}\}$. Then there is a subsequence $E^{\prime} \subset E$ such that $\# E^{\prime}=L\left(N_{2}\right)-R\left(N_{1}\right)+m$ and $E^{\prime}$ is also an $m$-spread sequence on $\left[t_{N_{1}}, t_{N_{2}}\right]$.

Proof. (i) Assume that $K:=\# E \geq L\left(N_{2}\right)-R\left(N_{1}\right)+m+1$. Let

$$
\begin{aligned}
& a_{n}=\#\left(E \cap\left[t_{N_{1}}, t_{n}\right)\right)-\left(R(n)-R\left(N_{1}\right)\right), \\
& b_{n}=\#\left(E \cap\left(t_{n}, t_{N_{2}}\right]\right)-\left(L\left(N_{2}\right)-L(n)\right), \quad N_{1} \leq n \leq N_{2} .
\end{aligned}
$$

Then we see from (1.5) and (1.6) that

$$
a_{n}, b_{n} \geq 0, \quad N_{1} \leq n \leq N_{2}
$$

Let

$$
\begin{aligned}
& n_{a}=\min \left\{n: N_{1} \leq n \leq N_{2} \quad \text { and } \quad a_{l} \geq 1, n \leq l \leq N_{2}\right\} \\
& n_{b}=\max \left\{n: N_{1} \leq n \leq N_{2} \quad \text { and } \quad b_{l} \geq 1, N_{1} \leq l \leq n\right\}
\end{aligned}
$$

(ii) Prove that $N_{1}+1 \leq n_{a} \leq n_{b}+1$.

Since $a_{N_{1}}=0$ and $a_{N_{2}} \geq K-1-\left(R\left(N_{2}\right)-R\left(N_{1}\right)\right) \geq L\left(N_{2}\right)-R\left(N_{2}\right)+m \geq 1$, thanks to (1.3), we have $N_{1}+1 \leq n_{a} \leq N_{2}$. By (2.2), we have $a_{n_{a}-1}=0$. Consequently,

$$
\#\left(E \cap\left[t_{N_{1}}, t_{n_{a}-1}\right)\right)=R\left(n_{a}-1\right)-R\left(N_{1}\right) .
$$

Therefore,

$$
\#\left(E \cap\left[t_{n_{a}-1}, t_{N_{2}}\right]\right)=K-\left(R\left(n_{a}-1\right)-R\left(N_{1}\right)\right) .
$$

For $N_{1} \leq n<L\left(n_{a}-1\right)$, we have $t_{n}<t_{n_{a}-1}$. By (1.7) and (2.4),

$$
\begin{aligned}
& \#\left(E \cap\left(t_{n}, t_{N_{2}}\right]\right) \\
= & \#\left(E \cap\left(t_{n}, t_{n_{a}-1}\right)\right)+\#\left(E \cap\left[t_{n_{a}-1}, t_{N_{2}}\right]\right) \\
\geq & \left(R\left(n_{a}-1\right)-L(n)-m\right)+\left(K-\left(R\left(n_{a}-1\right)-R\left(N_{1}\right)\right)\right) \\
= & K-L(n)+R\left(N_{1}\right)-m .
\end{aligned}
$$

Hence

$$
\begin{aligned}
b_{n} & =\#\left(E \cap\left(t_{n}, t_{N_{2}}\right]\right)-\left(L\left(N_{2}\right)-L(n)\right) \\
& \geq\left(K-L(n)+R\left(N_{1}\right)-m\right)-\left(L\left(N_{2}\right)-L(n)\right) \\
& =K-L\left(N_{2}\right)+R\left(N_{1}\right)-m \\
& \geq 1, \quad N_{1} \leq n<L\left(n_{a}-1\right) .
\end{aligned}
$$

For $L\left(n_{a}-1\right) \leq n \leq n_{a}-1$, we see from (2.4) that

$$
\begin{aligned}
b_{n} & =\#\left(E \cap\left(t_{n}, t_{N_{2}}\right]\right)-\left(L\left(N_{2}\right)-L(n)\right) \\
& =\#\left(E \cap\left(t_{n_{a}-1}, t_{N_{2}}\right]\right)-\left(L\left(N_{2}\right)-L\left(n_{a}-1\right)\right) \\
& \geq K-\left(R\left(n_{a}-1\right)-R\left(N_{1}\right)\right)-1-\left(L\left(N_{2}\right)-L\left(n_{a}-1\right)\right) \\
& \geq m-R\left(n_{a}-1\right)+L\left(n_{a}-1\right) \geq 1 .
\end{aligned}
$$

By (2.3), $n_{b} \geq n_{a}-1$.

(iii) Prove that $\#\left(E \cap\left(t_{n_{a}-1}, t_{n_{a}}\right)\right) \geq 1$. 
Observe that

$$
\begin{aligned}
\#\left(E \cap\left[t_{N_{1}}, t_{n_{a}}\right)\right) & \geq R\left(n_{a}\right)-R\left(N_{1}\right)+1 \geq R\left(n_{a}-1\right)-R\left(N_{1}\right)+1 \\
& =\#\left(E \cap\left[t_{N_{1}}, t_{n_{a}-1}\right)\right)+1 .
\end{aligned}
$$

We have $t_{n_{a}-1}<t_{n_{a}}$. Hence

$$
R\left(n_{a}-1\right)=n_{a}-1<n_{a}=L\left(n_{a}\right) \leq R\left(n_{a}\right) .
$$

By (2.2), $a_{n_{a}-1}=0$ and $a_{n} \geq 1$ for $n_{a} \leq n \leq N_{2}$. Hence

$$
\begin{aligned}
& \#\left(E \cap\left[t_{n_{a}-1}, t_{n}\right)\right) \\
= & \#\left(E \cap\left[t_{N_{1}}, t_{n}\right)\right)-\#\left(E \cap\left[t_{N_{1}}, t_{n_{a}-1}\right)\right) \\
\geq & \left(R(n)-R\left(N_{1}\right)+1\right)-\left(R\left(n_{a}-1\right)-R\left(N_{1}\right)\right) \\
= & R(n)-R\left(n_{a}-1\right)+1, \quad n_{a} \leq n \leq N_{2} .
\end{aligned}
$$

Therefore,

$$
\#\left(E \cap\left(t_{n_{a}-1}, t_{n}\right)\right) \geq R(n)-R\left(n_{a}-1\right), \quad n_{a} \leq n \leq N_{2} .
$$

By setting $n=n_{a}$, we get

$$
\#\left(E \cap\left(t_{n_{a}-1}, t_{n_{a}}\right)\right) \geq R\left(n_{a}\right)-R\left(n_{a}-1\right) \geq 1,
$$

thanks to (2.5).

(iv) Take some $y^{\prime} \in E \cap\left(t_{n_{a}-1}, t_{n_{a}}\right)$ and let $E^{\prime}=E \backslash\left\{y^{\prime}\right\}$. Then $E^{\prime}$ is an $m$-spread sequence on $\left[t_{N_{1}}, t_{N_{2}}\right]$.

Since \# $E^{\prime}=K-1 \geq L\left(N_{2}\right)-R\left(N_{1}\right)+m$, we only need to show that $E^{\prime}$ satisfies (1.5) -(1.7)

For $N_{1} \leq n \leq n_{a}-1$, we have

$$
\#\left(E^{\prime} \cap\left[t_{N_{1}}, t_{n}\right)\right)=\#\left(E \cap\left[t_{N_{1}}, t_{n}\right)\right)=a_{n}+\left(R(n)-R\left(N_{1}\right)\right) \geq R(n)-R\left(N_{1}\right) .
$$

For $n_{a} \leq n \leq N_{2}$, we have

$$
\#\left(E^{\prime} \cap\left[t_{N_{1}}, t_{n}\right)\right)=\#\left(E \cap\left[t_{N_{1}}, t_{n}\right)\right)-1=a_{n}+\left(R(n)-R\left(N_{1}\right)\right)-1 \geq R(n)-R\left(N_{1}\right) .
$$

On the other hand, for $N_{1} \leq n \leq n_{a}-1 \leq n_{b}$,

$$
\begin{aligned}
\#\left(E^{\prime} \cap\left(t_{n}, t_{N_{2}}\right]\right) & =\#\left(E \cap\left(t_{n}, t_{N_{2}}\right]\right)-1 \\
& =b_{n}+\left(L\left(N_{2}\right)-L(n)\right)-1 \geq L\left(N_{2}\right)-L(n) .
\end{aligned}
$$

For $n_{a} \leq n \leq N_{2}$, we have

$$
\begin{aligned}
\#\left(E^{\prime} \cap\left(t_{n}, t_{N_{2}}\right]\right) & =\#\left(E \cap\left(t_{n}, t_{N_{2}}\right]\right) \\
& =b_{n}+\left(L\left(N_{2}\right)-L(n)\right) \geq L\left(N_{2}\right)-L(n) .
\end{aligned}
$$

Hence (1.5) and (1.6) hold. Now it remains to prove that

$$
\#\left(E^{\prime} \cap\left(t_{n_{1}}, t_{n_{2}}\right)\right) \geq R\left(n_{2}\right)-L\left(n_{1}\right)-m, \quad \forall N_{1} \leq n_{1} \leq n_{2} \leq N_{2} .
$$

There are three cases.

Case 1. $n_{1} \geq n_{a}$ or $n_{2} \leq n_{a}-1$. In this case, we have

$$
\#\left(E^{\prime} \cap\left(t_{n_{1}}, t_{n_{2}}\right)\right)=\#\left(E \cap\left(t_{n_{1}}, t_{n_{2}}\right)\right) \geq R\left(n_{2}\right)-L\left(n_{1}\right)-m .
$$


Case 2. $n_{1}<L\left(n_{a}-1\right)$ and $n_{2} \geq n_{a}$. In this case,

$$
\begin{aligned}
\#\left(E^{\prime} \cap\left(t_{n_{1}}, t_{n_{2}}\right)\right) & =\#\left(E^{\prime} \cap\left(t_{n_{1}}, t_{n_{a}-1}\right)\right)+\#\left(E^{\prime} \cap\left[t_{n_{a}-1}, t_{n_{2}}\right)\right) \\
& =\#\left(E \cap\left(t_{n_{1}}, t_{n_{a}-1}\right)\right)+\#\left(E \cap\left[t_{n_{a}-1}, t_{n_{2}}\right)\right)-1 \\
& \geq\left(R\left(n_{a}-1\right)-L\left(n_{1}\right)-m\right)+\left(R\left(n_{2}\right)-R\left(n_{a}-1\right)\right) \\
& =R\left(n_{2}\right)-L\left(n_{1}\right)-m,
\end{aligned}
$$

thanks to (2.6).

Case 3. $L\left(n_{a}-1\right) \leq n_{1} \leq n_{a}-1$ and $n_{2} \geq n_{a}$. By (2.7), we have

$$
\begin{aligned}
\#\left(E^{\prime} \cap\left(t_{n_{1}}, t_{n_{2}}\right)\right) & =\#\left(E^{\prime} \cap\left(t_{n_{a}-1}, t_{n_{2}}\right)\right) \\
& =\#\left(E \cap\left(t_{n_{a}-1}, t_{n_{2}}\right)\right)-1 \\
& \geq R\left(n_{2}\right)-R\left(n_{a}-1\right)-1 \\
& =R\left(n_{2}\right)-R\left(n_{1}\right)-1 \\
& \geq R\left(n_{2}\right)-R\left(n_{1}\right)-m .
\end{aligned}
$$

Now, we get that $E^{\prime}$ is an $m$-spread sequence on $\left[t_{N_{1}}, t_{N_{2}}\right]$.

(v) Using the above procedures repeatedly, we obtain some $E^{\prime} \subset E$ such that $\# E^{\prime}=L\left(N_{2}\right)-R\left(N_{1}\right)+m$ and $E^{\prime}$ is an $m$-spread sequence on $\left[t_{N_{1}}, t_{N_{2}}\right]$.

Let $x_{1}<x_{2}<\cdots<x_{n}$ be real numbers and $f_{1}, f_{2}, \ldots, f_{n}$ be functions. Define

$$
M\left(\begin{array}{cc}
x_{1}, & x_{2}, \ldots, x_{n} \\
f_{1}, & f_{2}, \ldots, f_{n}
\end{array}\right)=\left[\begin{array}{llll}
f_{1}\left(x_{1}\right) & f_{2}\left(x_{1}\right) & \cdots & f_{n}\left(x_{1}\right) \\
f_{1}\left(x_{2}\right) & f_{2}\left(x_{2}\right) & \cdots & f_{n}\left(x_{2}\right) \\
& \ldots & \cdots & \\
f_{1}\left(x_{n}\right) & f_{2}\left(x_{n}\right) & \cdots & f_{n}\left(x_{n}\right)
\end{array}\right] .
$$

Lemma 2.2. Let $n, K$ be integers and $K>0$. For any real numbers $x_{0}<x_{1}<$ $\cdots<x_{K-1}$, the matrix $M\left(\begin{array}{cccc}x_{0} & x_{1} & \cdots & x_{K-1} \\ \varphi_{n} & \varphi_{n+1} & \cdots & \varphi_{n+K-1}\end{array}\right)$ is invertible if and only if

$$
\varphi_{n+k}\left(x_{k}\right) \neq 0, \quad 0 \leq k \leq K-1 .
$$

Proof. This is a consequence of [19, Theorem 4.61].

Lemma 2.3. Suppose that $E:=\left\{x_{k}: 0 \leq k \leq K-1\right\}$ is an $m$-spread sequence on $\left[t_{N_{1}}, t_{N_{2}}\right]$ with respect to $\left\{t_{k}: k \in \mathbb{Z}\right\}$, where $K=L\left(N_{2}\right)-R\left(N_{1}\right)+m$. Suppose that $x_{k-1}<x_{k}, 1 \leq k \leq K-1$. Then the matrix

$$
M\left(\begin{array}{cccc}
x_{0} & x_{1} & \cdots & x_{K-1} \\
\varphi_{R\left(N_{1}\right)-m} & \varphi_{R\left(N_{1}\right)-m+1} & \cdots & \varphi_{L\left(N_{2}\right)-1}
\end{array}\right)
$$

is invertible.

Proof. By Lemma 2.2, we only need to show that

$$
\varphi_{R\left(N_{1}\right)-m+k}\left(x_{k}\right) \neq 0, \quad 0 \leq k \leq K-1 .
$$

Assume that there is some $0 \leq k_{0} \leq K-1$ such that $\varphi_{R\left(N_{1}\right)-m+k_{0}}\left(x_{k_{0}}\right)=0$. Since $\varphi_{R\left(N_{1}\right)-m+k_{0}}(x)>0$ in $\left(t_{R\left(N_{1}\right)-m+k_{0}}, t_{R\left(N_{1}\right)+k_{0}+1}\right)$, there are only two cases.

Case 1. $x_{k_{0}} \leq t_{R\left(N_{1}\right)-m+k_{0}}$. Then we have

$$
t_{N_{1}} \leq x_{k} \leq x_{k_{0}} \leq t_{R\left(N_{1}\right)-m+k_{0}}, \quad 0 \leq k \leq k_{0} .
$$

Hence \# $\left(E \cap\left[t_{N_{1}}, t_{R\left(N_{1}\right)-m+k_{0}}\right]\right) \geq k_{0}+1$. On the other hand, since

$$
R\left(N_{1}\right)-m+k_{0} \leq R\left(N_{1}\right)-m+K-1=L\left(N_{2}\right)-1<N_{2},
$$


we see from (1.6) that

$$
\begin{aligned}
\#\left(E \cap\left(t_{R\left(N_{1}\right)-m+k_{0}}, t_{N_{2}}\right]\right) & \geq L\left(N_{2}\right)-L\left(R\left(N_{1}\right)-m+k_{0}\right) \\
& \geq L\left(N_{2}\right)-\left(R\left(N_{1}\right)-m+k_{0}\right) \\
& =K-k_{0} .
\end{aligned}
$$

Hence $\#\left(E \cap\left[t_{N_{1}}, t_{N_{2}}\right]\right) \geq K+1$, which contradicts $\# E=K$.

Case 2. $x_{k_{0}} \geq t_{R\left(N_{1}\right)+k_{0}+1}$. Then we have

$$
t_{R\left(N_{1}\right)+k_{0}+1} \leq x_{k_{0}} \leq x_{k} \leq t_{N_{2}}, \quad k_{0} \leq k \leq K-1 .
$$

Hence $\#\left(E \cap\left[t_{R\left(N_{1}\right)+k_{0}+1}, t_{N_{2}}\right]\right) \geq K-k_{0}$. But

$$
\#\left(E \cap\left[t_{N_{1}}, t_{R\left(N_{1}\right)+k_{0}+1}\right)\right) \geq R\left(R\left(N_{1}\right)+k_{0}+1\right)-R\left(N_{1}\right) \geq k_{0}+1,
$$

thanks to (1.5). Hence $\#\left(E \cap\left[t_{N_{1}}, t_{N_{2}}\right]\right) \geq K+1$. Again, this contradicts $\# E=$ K.

Proof of the sufficiency of Theorem 1.1. Assume that $E:=\left\{x_{k}: 0 \leq k \leq K-1\right\}$ is an $m$-spread sequence on $\left[t_{N_{1}}, t_{N_{2}}\right]$, where $x_{0}<x_{1}<\cdots<x_{K-1}$ and $K \geq K^{\prime}:=$ $L\left(N_{2}\right)-R\left(N_{1}\right)+m$. Let

$$
A=\left[\varphi_{n}\left(x_{k}\right)\right]_{0 \leq k \leq K-1, \quad R\left(N_{1}\right)-m \leq n \leq L\left(N_{2}\right)-1}
$$

be a $K \times K^{\prime}$ matrix. By Lemma 2.1 there is some subsequence $E^{\prime} \subset E$ which is also an $m$-spread sequence on $\left[t_{N_{1}}, t_{N_{2}}\right]$ such that $\# E^{\prime}=K^{\prime}$. Denote $E^{\prime}=$ $\left\{y_{k}: 0 \leq k \leq K^{\prime}-1\right\}$, where $y_{0}<y_{1}<\cdots<y_{K^{\prime}-1}$. By Lemma 2.3, we have

$$
A^{\prime}:=M\left(\begin{array}{cccc}
y_{0} & y_{1} & \cdots & y_{K^{\prime}-1} \\
\varphi_{R\left(N_{1}\right)-m} & \varphi_{R\left(N_{1}\right)-m+1} & \cdots & \varphi_{L\left(N_{2}\right)-1}
\end{array}\right)
$$

is invertible. Hence, there is some constant $\alpha>0$ such that

$$
\left\|A^{\prime} c\right\|_{2} \geq \alpha\|c\|_{2}, \quad \forall c \in \mathbb{C}^{K^{\prime}} .
$$

Since $A^{\prime}$ consists of $K^{\prime}$ rows of $A$, we have

$$
c^{T} A^{T} A c=\|A c\|_{2}^{2} \geq\left\|A^{\prime} c\right\|_{2}^{2} \geq \alpha^{2}\|c\|_{2}^{2}, \quad \forall c \in \mathbb{C}^{K^{\prime}} .
$$

Hence $A^{T} A$ is invertible.

For any $f \in V_{m}$, there are some $c_{n} \in \mathbb{C}$ such that $f(x)=\sum_{n \in \mathbb{Z}} c_{n} \varphi_{n}(x)$. Observe that $\operatorname{supp} \varphi_{n}=\left(t_{n}, t_{n+m+1}\right)$. We have supp $\varphi_{n} \subset\left(-\infty, t_{N_{1}}\right)$ for $n \leq R\left(N_{1}\right)-m-1$ and $\operatorname{supp} \varphi_{n} \subset\left(t_{N_{2}},+\infty\right)$ for $n \geq L\left(N_{2}\right)$. Hence $\varphi_{n}(x)=0$ for $x \in\left[t_{N_{1}}, t_{N_{2}}\right]$ and $n \notin\left[R\left(N_{1}\right)-m, L\left(N_{2}\right)-1\right]$. Therefore,

$$
f(x)=\sum_{n \in \mathbb{Z}} c_{n} \varphi_{n}(x)=\sum_{n=R\left(N_{1}\right)-m}^{L\left(N_{2}\right)-1} c_{n} \varphi_{n}(x), \quad \forall x \in\left[t_{N_{1}}, t_{N_{2}}\right] .
$$

It follows that

$$
F=A c,
$$

where

$$
F=\left[f\left(x_{0}\right), f\left(x_{1}\right), \ldots, f\left(X_{K-1}\right)\right]^{T}
$$

and

$$
c=\left[c_{R\left(N_{1}\right)-m}, c_{R\left(N_{1}\right)-m+1}, \ldots, c_{L\left(N_{2}\right)-1}\right]^{T} .
$$


Hence

$$
c=\left(A^{T} A\right)^{-1} A^{T} F
$$

Let

$$
\Phi=\left[\varphi_{R\left(N_{1}\right)-m}, \varphi_{R\left(N_{1}\right)-m+1}, \ldots, \varphi_{L\left(N_{2}\right)-1}\right]^{T}
$$

and

$$
\left[S_{0}, S_{1}, \ldots, S_{K-1}\right]^{T}=A\left(A^{T} A\right)^{-1} \Phi
$$

Then we have

$$
f(x)=c^{T} \Phi(x)=\sum_{k=0}^{K-1} f\left(x_{k}\right) S_{k}(x), \quad \forall x \in\left[t_{N_{1}}, t_{N_{2}}\right] .
$$

Obviously, supp $S_{k} \subset\left[t_{R\left(N_{1}\right)-m}, t_{L\left(N_{2}\right)+m}\right]$ since $\operatorname{supp} \varphi_{n}=\left(t_{n}, t_{n+m+1}\right)$.

Let $T_{\left[t_{N_{1}}, t_{N_{2}}\right]} f$ be the restriction of a function $f$ on $\left[t_{N_{1}}, t_{N_{2}}\right]$, i.e., $\left(T_{\left[t_{N_{1}}, t_{N_{2}}\right]} f\right)(x)$ $=f(x) \chi_{\left[t_{N_{1}}, t_{N_{2}}\right]}(x)$. Set

$$
V_{N_{1}, N_{2}}=T_{\left[t_{N_{1}}, t_{N_{2}}\right]} V_{m}:=\left\{T_{\left[t_{N_{1}}, t_{N_{2}}\right]} f: f \in V_{m}\right\} .
$$

Lemma 2.4. $\left\{T_{\left[t_{N_{1}}, t_{N_{2}}\right]} \varphi_{n}: R\left(N_{2}\right)-m \leq n \leq L\left(N_{2}\right)-1\right\}$ is a basis for $V_{N_{1}, N_{2}}$.

Proof. First, for any $f \in V_{N_{1}, N_{2}}$, there are constants $c_{R\left(N_{1}\right)-m}, c_{R\left(N_{1}\right)-m+1}, \ldots$, $c_{L\left(N_{2}\right)-1}$ such that

$$
f(x)=\sum_{n=R\left(N_{1}\right)-m}^{L\left(N_{2}\right)-1} c_{n} \varphi_{n}(x)=\sum_{n=R\left(N_{1}\right)-m}^{L\left(N_{2}\right)-1} c_{n} T_{\left[t_{N_{1}}, t_{N_{2}}\right]} \varphi_{n}(x), \quad x \in\left[t_{N_{1}}, t_{N_{2}}\right] .
$$

Hence it suffices to show that $\left\{T_{\left[t_{N_{1}}, t_{N_{2}}\right]} \varphi_{n}: R\left(N_{1}\right)-m \leq n \leq L\left(N_{2}\right)-1\right\}$ is linearly independent, or equivalently, $f(x)=0$ on $\left[t_{N_{1}}, t_{N_{2}}\right]$ implies that $c_{n}=$ $0, R\left(N_{1}\right)-m \leq n \leq L\left(N_{2}\right)-1$.

To see this, take $2 m$ points in each interval of the form $\left(t_{L(n)}, t_{R(n)+1}\right), n \geq N_{1}$. Let $E$ be the collection of all these points. It is easy to see that $E$ is a local sampling sequence on $\left[t_{N_{1}}, t_{N_{2}}\right]$ with respect to $\left\{t_{k}: k \in \mathbb{Z}\right\}$. Let $A$ be defined as in (2.8). Then we see from (2.11) that

$$
c_{n}=0, \quad R\left(N_{1}\right)-m \leq n \leq L\left(N_{2}\right)-1,
$$

since $f\left(x_{k}\right)=0,0 \leq k \leq K-1$.

Proof of the necessity of Theorem 1.1. Suppose that $E=\left\{x_{k}: 0 \leq k \leq K-1\right\}$ is a local sampling sequence for $V_{m}$ on $\left[t_{N_{1}}, t_{N_{2}}\right]$, where $x_{k-1}<x_{k}, 1 \leq k \leq K-1$. Then there is some sequence of functions $\left\{S_{k}: 0 \leq k \leq K-1\right\}$ such that (1.8) holds.

For any $f \in V_{N_{1}, N_{2}}, f(x)=\sum_{k=0}^{K-1} f\left(x_{k}\right) S_{k}(x)$ on $\left[t_{N_{1}}, t_{N_{2}}\right]$. Hence $\operatorname{dim} V_{N_{1}, N_{2}}$ $\leq K$. On the other hand, we see from Lemma 2.4 that $\operatorname{dim} V_{N_{1}, N_{2}}=L\left(N_{2}\right)-$ $R\left(N_{1}\right)+m$. Hence $K \geq L\left(N_{2}\right)-R\left(N_{1}\right)+m$.

Next we prove that (1.5) holds. Otherwise, there is some $N_{1}+1 \leq n_{0} \leq N_{2}$ such that $\#\left(E \cap\left[t_{N_{1}}, t_{n_{0}}\right)\right)<R\left(n_{0}\right)-R\left(N_{1}\right)$. Then we have $R\left(n_{0}\right) \geq R\left(N_{1}\right)+1$ and $x_{R\left(n_{0}\right)-R\left(N_{1}\right)-1} \geq t_{n_{0}}$. Consequently,

$$
\varphi_{n}\left(x_{R\left(n_{0}\right)-R\left(N_{1}\right)-1}\right)=0, \quad \forall R\left(N_{1}\right)-m \leq n \leq R\left(n_{0}\right)-m-1,
$$


thanks to $\operatorname{supp} \varphi_{n}=\left(t_{n}, t_{n+m+1}\right)$. Therefore, the last row of the matrix

$$
M\left(\begin{array}{cccc}
x_{0} & x_{1} & \cdots & x_{R\left(n_{0}\right)-R\left(N_{1}\right)-1} \\
\varphi_{R\left(N_{1}\right)-m} & \varphi_{R\left(N_{1}\right)-m+1} & \cdots & \varphi_{R\left(n_{0}\right)-m-1}
\end{array}\right)
$$

is 0 . Hence its rank is no greater than $R\left(n_{0}\right)-R\left(N_{1}\right)-1<R\left(n_{0}\right)-R\left(N_{1}\right)$. Therefore, we can find some $c_{R\left(N_{1}\right)-m}, c_{R\left(N_{1}\right)-m+1}, \ldots, c_{R\left(n_{0}\right)-m-1}$, not all of which are zeros, such that

$$
\sum_{l=R\left(N_{1}\right)-m}^{R\left(n_{0}\right)-m-1} c_{l} \varphi_{l}\left(x_{k}\right)=0, \quad 0 \leq k \leq R\left(n_{0}\right)-R\left(N_{1}\right)-1 .
$$

Let

$$
f(x)=\sum_{l=R\left(N_{1}\right)-m}^{R\left(n_{0}\right)-m-1} c_{l} \varphi_{l}(x) .
$$

Then we have $f\left(x_{k}\right)=0,0 \leq k \leq R\left(n_{0}\right)-R\left(N_{1}\right)-1$. On the other hand, since supp $f \subset\left(-\infty, t_{R\left(n_{0}\right)}\right)=\left(-\infty, t_{n_{0}}\right)$ and $x_{k} \geq x_{R\left(n_{0}\right)-R\left(N_{1}\right)-1} \geq t_{n_{0}}$ for $k \geq R\left(n_{0}\right)-R\left(N_{1}\right)-1$, we also have $f\left(x_{k}\right)=0$ for $k \geq R\left(n_{0}\right)-R\left(N_{1}\right)-1$. Hence

$$
f\left(x_{k}\right)=0, \quad 0 \leq k \leq K-1 .
$$

It follows that we cannot reconstruct $f$ on $\left[t_{N_{1}}, t_{N_{2}}\right]$ from $\left\{f\left(x_{k}\right): 0 \leq k \leq K-1\right\}$ since, by virtue of Lemma 2.4 $f$ is not identically 0 on $\left[t_{N_{1}}, t_{N_{2}}\right]$. This contradicts the hypothesis.

Similarly, we can prove that (1.6) holds.

Now, it remains to establish (1.7). If (1.7) does not hold, then there are some $N_{1} \leq n_{1}<n_{2} \leq N_{2}$ such that $\#\left(E \cap\left(t_{n_{1}}, t_{n_{2}}\right)\right)<R\left(n_{2}\right)-L\left(n_{1}\right)-m$. Then we have $R\left(n_{2}\right)-L\left(n_{1}\right)-m \geq 1$. Therefore,

$$
R\left(n_{2}\right)-L\left(n_{1}\right) \geq m+1
$$

There are two cases.

Case 1. $\#\left(E \cap\left(t_{n_{1}}, t_{n_{2}}\right)\right)=0$. In this case, we have

$$
x_{k} \leq t_{n_{1}} \quad \text { or } \quad x_{k} \geq t_{n_{2}}, \quad 0 \leq k \leq K-1 .
$$

Let $f=\varphi_{L\left(n_{1}\right)}$. Then we have supp $f=\left(t_{L\left(n_{1}\right)}, t_{L\left(n_{1}\right)+m+1}\right) \subset\left(t_{L\left(n_{1}\right)}, t_{R\left(n_{2}\right)}\right)=$ $\left(t_{n_{1}}, t_{n_{2}}\right)$. By (2.15), $f\left(x_{k}\right)=0,0 \leq k \leq K-1$. Since $E$ is a local sampling sequence, we have $f=0$ on $\left[t_{N_{1}}, t_{N_{2}}\right]$, which is impossible since $t_{L\left(n_{1}\right)+m+1}-$ $t_{L\left(n_{1}\right)}>0$ and $f>0$ in $\left(t_{L\left(n_{1}\right)}, t_{L\left(n_{1}\right)+m+1}\right)$.

Case 2. $\#\left(E \cap\left(t_{n_{1}}, t_{n_{2}}\right)\right) \geq 1$.

Let

$$
\begin{aligned}
& k_{1}=\min \left\{k: 0 \leq k \leq K-1, x_{k} \in E \cap\left(t_{n_{1}}, t_{n_{2}}\right)\right\}, \\
& k_{2}=\max \left\{k: 0 \leq k \leq K-1, x_{k} \in E \cap\left(t_{n_{1}}, t_{n_{2}}\right)\right\} .
\end{aligned}
$$

Denote the $\left(K-k_{1}\right) \times\left(L\left(N_{2}\right)-L\left(n_{1}\right)\right)$ matrix $\left[\varphi_{l}\left(x_{k}\right)\right]_{k_{1} \leq k \leq K-1, L\left(n_{1}\right) \leq l \leq L\left(N_{2}\right)-1}$ by $A=\left(\begin{array}{c}A_{1} \\ A_{2}\end{array}\right)$, where

$$
A_{1}=\left[\varphi_{l}\left(x_{k}\right)\right]_{k_{1} \leq k \leq k_{2}, L\left(n_{1}\right) \leq l \leq L\left(N_{2}\right)-1}
$$


and

$$
A_{2}=\left[\varphi_{l}\left(x_{k}\right)\right]_{k_{2}+1 \leq k \leq K-1, L\left(n_{1}\right) \leq l \leq L\left(N_{2}\right)-1} .
$$

Since there are $k_{2}-k_{1}+1 \leq R\left(n_{2}\right)-L\left(n_{1}\right)-m-1$ rows in $A_{1}$, we have

$$
\operatorname{rank}\left(A_{1}\right) \leq R\left(n_{2}\right)-L\left(n_{1}\right)-m-1 \text {. }
$$

Observe that

$$
\operatorname{supp} \varphi_{l} \subset\left(-\infty, t_{n_{2}}\right), \quad l \leq R\left(n_{2}\right)-m-1 .
$$

We have $\varphi_{l}\left(x_{k}\right)=0$ for $l \leq R\left(n_{2}\right)-m-1$ and $x_{k} \geq t_{n_{2}}$. Hence the first $R\left(n_{2}\right)-$ $L\left(n_{1}\right)-m$ columns of $A_{2}$ are 0 . Thus

$\operatorname{rank}\left(A_{2}\right) \leq\left(L\left(N_{2}\right)-L\left(n_{1}\right)\right)-\left(R\left(n_{2}\right)-L\left(n_{1}\right)-m\right)=L\left(N_{2}\right)-R\left(n_{2}\right)+m$.

Therefore,

$\operatorname{rank}(A) \leq \operatorname{rank}\left(A_{1}\right)+\operatorname{rank}\left(A_{2}\right) \leq L\left(N_{2}\right)-L\left(n_{1}\right)-1<L\left(N_{2}\right)-L\left(n_{1}\right)$.

Thus, we can find some $c_{L\left(n_{1}\right)}, c_{L\left(n_{1}\right)+1}, \ldots, c_{L\left(N_{2}\right)-1}$, not all of which are zeros, such that

$$
\sum_{l=L\left(n_{1}\right)}^{L\left(N_{2}\right)-1} c_{l} \varphi_{l}\left(x_{k}\right)=0, \quad k_{1} \leq k \leq K-1 .
$$

Let

$$
f(x)=\sum_{l=L\left(n_{1}\right)}^{L\left(N_{2}\right)-1} c_{l} \varphi_{l}(x) .
$$

Then we have $f\left(x_{k}\right)=0, k_{1} \leq k \leq K-1$. On the other hand, since $x_{k} \leq t_{n_{1}}$ for $0 \leq k \leq k_{1}-1$ and supp $f \subset\left(t_{n_{1}},+\infty\right)$, we also have $f\left(x_{k}\right)=0$ for $0 \leq k \leq k_{1}-1$. Now we get

$$
f\left(x_{k}\right)=0, \quad 0 \leq k \leq K-1 .
$$

Hence, we cannot reconstruct $f$ on $\left[t_{N_{1}}, t_{N_{2}}\right]$ from $\left\{f\left(x_{k}\right): 0 \leq k \leq K-1\right\}$ since $f \neq 0$, thanks to Lemma 2.4. Again, we get a contradiction. This completes the proof.

Proof of Corollary 1.2, It suffices to show that, under the assumption $t_{n}=\lfloor n / r\rfloor$, conditions (1.9) -(1.12) are equivalent to (1.4)-(1.7).

Observe that for $n=r N+l, 0 \leq l \leq r-1$,

$$
L(n)=r N \quad \text { and } \quad R(n)=r N+r-1 .
$$

First, we assume that (1.4)-1.7) hold. Set $N_{1}=r N_{1}^{\prime}$ and $N_{2}=r N_{2}^{\prime}$. Then we have $t_{N_{1}}=N_{1}^{\prime}$ and $t_{N_{2}}=N_{2}^{\prime}$. By (1.4),

$$
\# E \geq L\left(N_{2}\right)-R\left(N_{1}\right)+m=r\left(N_{2}^{\prime}-N_{1}^{\prime}-1\right)+m+1 .
$$

For $0 \leq k \leq N_{2}^{\prime}-N_{1}^{\prime}$, we have

$$
\begin{aligned}
\#\left(E \cap\left[N_{1}^{\prime}, N_{1}^{\prime}+k\right)\right) & =\#\left(E \cap\left[t_{r N_{1}^{\prime}}, t_{r N_{2}^{\prime}+r k}\right)\right) \\
& \geq R\left(r N_{2}^{\prime}+r k\right)-R\left(r N_{1}^{\prime}\right)=r k .
\end{aligned}
$$

Similarly, we can prove that

$$
\#\left(E \cap\left(N_{2}^{\prime}-k, N_{2}^{\prime}\right]\right)=\#\left(E \cap\left[t_{r N_{2}^{\prime}-r k}, t_{r N_{2}^{\prime}+r-1}\right)\right) \geq r k
$$

and

$$
\#\left(E \cap\left(n_{1}^{\prime}, n_{2}^{\prime}\right)\right)=\#\left(E \cap\left(t_{r n_{1}^{\prime}}, t_{r n_{2}^{\prime}}\right)\right) \geq r\left(n_{2}^{\prime}-n_{1}^{\prime}+1\right)-m-1
$$


Next we prove the converse. Fix some $N_{1}^{\prime}<N_{2}^{\prime}$. Observe that

$$
t_{r n-1}<t_{r n}=t_{r n+1}=\cdots=t_{r n+r-1}<t_{r(n+1)}, \quad \forall n \in \mathbb{Z} .
$$

We prove that (1.4) - (1.7) hold for $N_{1}=r N_{1}^{\prime}+l_{1}$ and $N_{2}=r N_{2}^{\prime}+l_{2}, 0 \leq l_{1}, l_{2} \leq$ $r-1$. By (1.9),

$$
\begin{aligned}
\# E & \geq r\left(N_{2}^{\prime}-N_{1}^{\prime}-1\right)+m+1=r N_{2}^{\prime}-\left(r N_{1}^{\prime}+r-1\right)+m \\
& =L\left(N_{2}\right)-R\left(N_{1}\right)+m .
\end{aligned}
$$

On the other hand, for $n=r N_{1}^{\prime}+r k+l, 0 \leq k \leq N_{2}^{\prime}-N_{1}^{\prime}$ and $0 \leq l \leq r-1$, we have

$$
\#\left(E \cap\left[t_{N_{1}}, t_{n}\right)\right)=\#\left(E \cap\left[N_{1}^{\prime}, N_{1}^{\prime}+k\right)\right) \geq r k=R(n)-R\left(N_{1}\right) .
$$

Similarly, we can show that for $n=r N_{2}^{\prime}-r k+l, 0 \leq k \leq N_{2}^{\prime}-N_{1}^{\prime}$ and $0 \leq l \leq r-1$,

$$
\#\left(E \cap\left(t_{n}, t_{N_{2}}\right]\right) \geq L\left(N_{2}\right)-L(n) .
$$

Finally, for $n_{1}=r N_{1}^{\prime}+r n_{1}^{\prime}+l_{1}^{\prime}$ and $n_{2}=r N_{2}^{\prime}+r n_{2}^{\prime}+l_{2}^{\prime}$, where $0 \leq l_{1}^{\prime}, l_{2}^{\prime} \leq r-1$, we have

$$
\begin{aligned}
\#\left(E \cap\left[t_{n_{1}}, t_{n_{2}}\right)\right) & =\#\left(E \cap\left(N_{1}^{\prime}+n_{1}^{\prime}, N_{1}^{\prime}+n_{2}^{\prime}\right)\right) \\
& \geq r n_{2}^{\prime}-r n_{1}^{\prime}+r-m-1=R\left(n_{2}\right)-L\left(n_{1}\right)-m .
\end{aligned}
$$

This completes the proof.

\section{LOCAL RECONSTRUCTION OF FUNCTIONS FROM FINITELY MANY SAMPLES}

In this section, we give some applications of Theorem 1.1. We show that under certain conditions, we can reconstruct a function on an interval using only a finite number of samples. For simplicity, we consider the function space $V_{m}^{(r)}$ generated by $m$-degree normalized B-splines $\psi_{l}$ with knots $(\lfloor l / r\rfloor,\lfloor(l+1) / r\rfloor, \ldots,\lfloor(l+m+1) / r\rfloor)$, $l \in \mathbb{Z}$, where $1 \leq r \leq m$ are fixed integers.

3.1. Irregular sampling. Irregular sampling is widely studied in literature. For functions in the space $V_{m}^{(1)}$ generated by the $m$-degree cardinal B-splines, Aldroubi and Gröchening 2 proved that if the sampling sequence $\left\{x_{k}: k \in \mathbb{Z}\right\}$ satisfies

$$
0<\alpha \leq x_{k+1}-x_{k} \leq \beta<1, \quad k \in \mathbb{Z},
$$

then there are constants $C_{1}, C_{2}>0$ such that

$$
C_{1}\|f\|_{p}^{p} \leq \sum_{k \in \mathbb{Z}}\left|f\left(x_{k}\right)\right|^{p} \leq C_{2}\|f\|_{p}^{p}, \quad \forall f \in V_{m}^{(1)} \cap L^{p}(\mathbb{R})
$$

and every $f \in V_{m}^{(1)} \cap L^{p}(\mathbb{R})$ can be reconstructed from $\left\{f\left(x_{k}\right): k \in \mathbb{Z}\right\}$. The standard procedure to reconstruct a function from sampled values is carried out with the frame algorithm [2, 27.

In this subsection, we give a similar result for $V_{m}^{(r)}$. Moreover, our approach allows us to reconstruct a function locally using only finitely many samples.

Theorem 3.1. Let $\left\{x_{k}: k \in \mathbb{Z}\right\} \subset \mathbb{R}$ be a sequence such that

$$
x_{k+1}-x_{k}>0 \text { and } x_{k+r}-x_{k} \leq \beta<1, \quad k \in \mathbb{Z} \text {. }
$$

Then for any integers $N_{1}<N_{2}$, there are finitely many functions $S_{k}, k_{1} \leq k \leq k_{2}$, such that

$$
f(x)=\sum_{k_{1} \leq k \leq k_{2}} f\left(x_{k}\right) S_{k}(x), \quad \forall f \in V_{m}^{(r)}, x \in\left[N_{1}, N_{2}\right] .
$$


Proof. Denote $E=\left\{x_{k}: k \in \mathbb{Z}\right\}$. Take some integer $N_{2}^{\prime}$ such that $N_{2}^{\prime}-N_{1} \geq$ $(m+1) \beta /(r(1-\beta))$. Let $k_{1}=\min \left\{k \in \mathbb{Z}: x_{k} \geq N_{1}\right\}$ and $k_{2}=$ $\max \left\{k \in \mathbb{Z}: x_{k} \leq N_{2}^{\prime}\right\}$.

Since $x_{k+r}-x_{k} \leq \beta$, there are at least $r$ points of $E$ in every interval of length $\beta$. It follows that

$$
\#\left(E \bigcap\left[N_{1}, N_{2}^{\prime}\right]\right) \geq\left\lfloor\frac{N_{2}^{\prime}-N_{1}}{\beta}\right\rfloor \cdot r \geq\left(\frac{N_{2}^{\prime}-N_{1}}{\beta}-1\right) \cdot r .
$$

Since $N_{2}^{\prime}-N_{1} \geq(m+1) \beta /(r(1-\beta))$, we have $\#\left(E \bigcap\left[N_{1}, N_{2}^{\prime}\right]\right) \geq r\left(N_{2}^{\prime}-N_{1}-1\right)+$ $m+1$. On the other hand, it is easy to see that $E$ satisfies (1.10), (1.11) and (1.12). By Corollary 1.2, $E$ is a local sampling sequence for $V_{m}^{(r)}$ on $\left[N_{1}, N_{2}^{\prime}\right]$. Similarly to (2.13) we can find functions $S_{k}, k_{1} \leq k \leq k_{2}$, such that

$$
f(x)=\sum_{k_{1} \leq k \leq k_{2}} f\left(x_{k}\right) S_{k}(x), \quad \forall f \in V_{m}^{(r)}, x \in\left[N_{1}, N_{2}\right] .
$$

Remark 3.1. If $E:=\left\{x_{k}: k \in \mathbb{Z}\right\}$ is a sampling sequence for $V_{m}^{(r)}$, i.e., there are functions $S_{k}$ such that

$$
f(x)=\sum_{k \in \mathbb{Z}} f\left(x_{k}\right) S_{k}(x), \quad \forall f \in V_{m}^{(r)},
$$

then we see from the proof of the necessity of Theorem 1.1 that for any integers $n_{1}<n_{2}$,

$$
\#\left(E \cap\left[n_{1}, n_{2}\right]\right) \geq r\left(n_{2}-n_{1}+1\right)-m-1 .
$$

Hence

$$
\limsup _{\left(n_{2}-n_{1}\right) \rightarrow \infty} \frac{\#\left(E \cap\left[n_{1}, n_{2}\right]\right)}{n_{2}-n_{1}} \geq r .
$$

That is, the average density of sampling points is $r$. Therefore, the hypothesis $x_{k+r}-x_{k} \leq \beta<1$ in Theorem 3.1 is reasonable.

3.2. Average sampling. Average sampling theorems for shift invariant subspaces have been established recently, e.g., see [1, 2, 21, 22, 23, 27] and references therein. Again, all of these results consider global sampling. That is, to recover a function on a bounded interval, we have to know all the averages near every sampling points.

In [10, Theorem 8.15], Feichtinger and Gröchenig proved that if $\delta:=$ $\sup _{k \in \mathbb{Z}}\left(x_{k+1}-x_{k}\right)<\frac{\pi}{\Omega}$, then every $f$ with band limited to $[-\Omega, \Omega]$ is uniquely determined by averages $\frac{1}{y_{k}-y_{k-1}} \int_{y_{k-1}}^{y_{k}} f(x) d x$, where $y_{k}=\frac{x_{k}+x_{k+1}}{2}, k \in \mathbb{Z}$.

In this paper, we give a similar result for the space $V_{m}^{(r)}$. Moreover, we prove that the standard averaging function can be replaced by any other one which is supported in $\left[y_{k-1}, y_{k}\right)$. Again, our approach allows us to reconstruct a function on a finite interval using only finitely many samples.

Definition 3.1. We call $u(x)$ an averaging function if $u(x) \geq 0$ and $\int_{\mathbb{R}} u(x) d x=1$.

Theorem 3.2. Suppose that $N_{1}=x_{0}<x_{1}<\cdots<x_{K}=N_{2}$ and

$$
x_{k+r}-x_{k}<1, \quad 0 \leq k \leq K-r,
$$

where $K \geq r\left(N_{2}-N_{1}-1\right)+m+1$. Let $\left\{u_{k}: 0 \leq k \leq K-1\right\}$ be a sequence of averaging functions such that $\operatorname{supp} u_{k} \subset\left[x_{k}, x_{k+1}\right), 0 \leq k \leq K-1$. Then there 
exist functions $S_{k} \in V_{m}^{(r)}$ such that

$$
f(x)=\sum_{k=0}^{K-1}\left\langle f, u_{k}\right\rangle S_{k}(x), \quad \forall f \in V_{m}^{(r)}, \quad x \in\left[N_{1}, N_{2}\right] .
$$

Proof. Fix some real sequence $\left\{c_{l}: l \in \mathbb{Z}\right\}$ and let $f(x)=\sum_{l \in \mathbb{Z}} c_{l} \psi_{l}(x)$.

Using the mean value theorem, for any $0 \leq k \leq K-1$, we can find some $y_{k} \in \operatorname{supp} u_{k} \subset\left[x_{k}, x_{k+1}\right)$ such that

$$
\left\langle f, u_{k}\right\rangle=f\left(y_{k}\right)
$$

Using the hypotheses, it is easy to check that the point sequence $\left\{y_{k}: 0 \leq k \leq\right.$ $K-1$ \} meets Corollary 1.2, Let

$$
A=\left[\psi_{l}\left(y_{k}\right)\right]_{0 \leq k \leq K-1, r+r N_{1}-m-1 \leq l \leq r N_{2}-1}
$$

be a $K \times\left(r\left(N_{2}-N_{1}-1\right)+m\right)$ matrix. We see from the proof of the sufficiency of Theorem 1.1 that $A^{T} A$ is invertible. Observe that

$$
f\left(y_{k}\right)=\sum_{r+r N_{1}-m-1 \leq l \leq r N_{2}-1} c_{l} \psi_{l}\left(y_{k}\right)=\sum_{r+r N_{1}-m-1 \leq l \leq r N_{2}-1} c_{l}\left\langle\psi_{l}, u_{k}\right\rangle .
$$

We have

$$
\sum_{0 \leq k \leq K-1}\left|f\left(y_{k}\right)\right|^{2}=c^{T} A^{T} A c=c^{T} B^{T} B c,
$$

where $c=\left[c_{r+r N_{1}-m-1}, c_{r+r N_{1}-m}, \ldots, c_{r N_{2}-1}\right]^{T}$ and

$$
B=\left[\left\langle\psi_{l}, u_{k}\right\rangle\right]_{0 \leq k \leq K-1, r+r N_{1}-m-1 \leq l \leq r N_{2}-1} .
$$

Note that $y_{k}$, and therefore $A$ depends on the coefficients $c_{l}$. Nevertheless, since $A^{T} A$ is invertible, we see from (3.4) that

$$
c^{T} B^{T} B c>0, \quad \forall c \in \mathbb{R}^{r\left(N_{2}-N_{1}-1\right)+m} \backslash\{0\} .
$$

Hence $B^{T} B$ is also invertible.

Let $F=\left[\left\langle f, u_{0}\right\rangle,\left\langle f, u_{1}\right\rangle, \ldots,\left\langle f, u_{K-1}\right\rangle\right]^{T}$. Then we have $F=B c$ and

$$
c=\left(B^{T} B\right)^{-1} B^{T} F .
$$

Define $\left[S_{0}, S_{1}, \ldots, S_{K-1}\right]^{T}=B\left(B^{T} B\right)^{-1} \Psi$, where

$$
\Psi=\left[\psi_{r+r N_{1}-m-1}, \psi_{r+r N_{1}-m}, \ldots, \psi_{r N_{2}-1}\right]^{T} .
$$

Then we have

$$
f(x)=c^{T} \Psi(x)=\sum_{k=0}^{K-1}\left\langle f, u_{k}\right\rangle S_{k}(x), \quad \forall x \in\left[N_{1}, N_{2}\right] .
$$

This completes the proof.

The following is an immediate consequence. 
Corollary 3.3. Suppose that $N_{1}=x_{0}<x_{1}<\cdots<x_{K}=N_{2}$ and

$$
x_{k+r}-x_{k}<1, \quad 0 \leq k \leq K-r,
$$

where $K \geq r\left(N_{2}-N_{1}-1\right)+m+1$. Then there exist functions $S_{k} \in V_{m}^{(r)}$ such that

$$
f(x)=\sum_{k=0}^{K-1} \frac{1}{x_{k+1}-x_{k}} \int_{x_{k}}^{x_{k+1}} f(t) d t S_{k}(x), \quad \forall f \in V_{m}^{(r)}, \quad x \in\left[N_{1}, N_{2}\right] .
$$

Remark 3.2. The point sequence $x_{k}=N_{1}+\left(N_{2}-N_{1}\right) k / K, 0 \leq k \leq K$, satisfies the hypotheses of Theorem 3.2 and Corollary 3.3 .

\section{ACKNOWLEDGEMENTS}

The author thanks the referee for many useful suggestions which helped to improve the paper.

\section{REFERENCES}

[1] A. Aldroubi, Non-uniform weighted average sampling and reconstruction in shiftinvariant and wavelet spaces, Appl. Comp. Harmonic Anal., 13(2002), 151-161. MR.1942749 (2003i:42045)

[2] A. Aldroubi and K. Gröchenig, Beuling-Landau-type theorems for non-uniform sampling in shift invariant spline spaces, J. Fourier Anal. Appl., 6 (2000), 93-103. MR 1756138 (2001i:42057)

[3] A. Aldroubi and M. Unser, Sampling procedures in function spaces and asymptotic equivalence with Shannon's sampling theory, Numer. Funct. Anal. and Optimiz., 15 (1994), 1-21. MR.1261594 (95a:94002)

[4] N. Atreas, J.J. Benedetto and C. Karanikas, Local sampling for regular wavelet and Gabor expansions, Sampl. Theory Signal Image Process, 2(2003), 1-24. MR.2002854 (2004k:42050)

[5] A. Beurling, in L. Carleson, Ed., A. Beurling Collected Works, Vol. 2, Birkhäuser, Boston, 1989, 341-365.

[6] P.L. Butzer and J. Lei, Approximation of signals using measured sampled values and error analysis, Commun. Appl. Anal., 4(2000), 245-255. MR1752849 (2001i:94033)

[7] O. Christensen, An Introduction to Frames and Riesz Bases, Birkhäuser, Boston, 2003. MR.1946982 (2003k:42001)

[8] C.K. Chui, An Introduction to Wavelets, Academic Press, New York, 1992. MR1150048 (93f:42055)

[9] I. Daubechies, Ten Lectures on Wavelets, SIAM Philadelphia, 1992. MR.1162107(93e:42045)

[10] H. Feichtinger and K. Gröchenig, Theory and practice of irregular sampling, in "Wavelets: Mathematics and Applications" (J. Benedetto and M. Frazier, eds.), 305-363, CRC press Inc., 1994. MR 1247520(94i:94008)

[11] T.N. Goodman and S.L. Lee, Wavelets of multiplicity $r$, Trans. Amer. Math. Soc., 342(1994), 307-324. MR1232187 (94k:41016)

[12] K. Gröchenig, Reconstruction algorithms in irregular sampling, Math. Comput., 59(1992), 181-194. MR1134729 (93a:41025)

[13] S. Jaffard, A density criterion for frames of complex expotentials, Michigan Math. J., 38(1991), 339-348. MR1116493 (92i:42001)

[14] A.J.E.M. Janssen, The Zak transform and sampling theorem for wavelet subspaces, IEEE Trans. Signal processing, 41 (1993), 3360-3364.

[15] H. Landau, Necessary density conditions for sampling and interpolation of certain entire functions, ACTA Math., 117(1967), 37-52. MR0222554 (36:5604)

[16] Y. Liu, Irregular sampling for spline wavelet subspaces, IEEE Trans. Inform. Theory, 42 (1996), 623-627. MR1381369 (97b:94006)

[17] P.R. Massopust, D.K. Ruch And P.J. Van Fleet, On the support properties of scaling vectors, Appl. Comput. Harmon. Anal., 3(1996), 229-238. MR1400081(97i:42018)

[18] J. Ortega-Cerdà and K. Seip, Fourier frames, Ann. of Math., 155(2002), 789-806. MR1923965 (2003k:42055) 
[19] L. Schumaker, Spline Functions: Basic Theory, Wiley-Interscience, Boston, 1981. MR606200 (82j:41001)

[20] C.E. Shannon, Communication in the presence of noise, Proc. IRE., 37(1949), 10-21. MR0028549 (10:464e)

[21] W. Sun, X. Zhou, Average sampling theorems for shift invariant subspaces, Sci. China Ser. E 43(2000) 524-530. MR1799696 (2001m:94030)

[22] W. Sun and X. Zhou, Average sampling in spline subspaces, Appl. Math. Letters, 15(2002) 233-237. MR1880763 (2003a:94026)

[23] W. Sun and X. Zhou, Reconstruction of functions in spline subspaces from local averages, Proc. Amer. Math. Soc. 131(2003) 2561-2571. MR1974656 (2004f:42057)

[24] W. Sun and X. Zhou, Characterization of local sampling sequences in spline subspaces, Adv. Comput. Math., to appear.

[25] G. Walter, A sampling theorem for wavelet subspaces, IEEE Trans. Inform. Theory, 38 (1992), 881-884. MR1162226 (93e:94006)

[26] R.G. Wiley, Recovery of band-limited signals from unequally spaced samples, IEEE Trans. Comm., 26 (1978), 135-137.

[27] J. Xian, Weighted sampling and signal reconstruction in spline subspaces, Signal Processing, 86(2006), 331-340.

[28] R.M. Young, An Introduction to Non-Harmonic Fourier Series, Academic, New York, 1980. MR591684 (81m:42027)

[29] C. Zhao and P. Zhao, Sampling theorem and irregular sampling theorem for multiwavelet subspaces, IEEE Trans. Signal Proc., 53(2005), 705-713. MR.2117324

[30] X. Zhou, W. Sun, On the sampling theorem for wavelet subspaces, J. Fourier Anal. Appl., $\mathbf{5}$ (1999), 347-354. MR1700088 (2000i:42025)

Department of Mathematics and LPMC, Nankai University, Tianjin 300071, China

E-mail address: sunwch@nankai.edu.cn 